\title{
BURNOUT E ENFRENTAMENTO EM JOGADORES DE FUTEBOL: FASES PRÉ E DURANTE COMPETIÇÃO
}

\author{
BURNOUT AND COPING AMONG FOOTBALL PLAYERS: BEFORE AND DURING THE TOURNAMENT
}

TEXERC10

BURNOUT Y ENFRENTAMIENTO EN JUGADORES DE FÚTBOL: FASES PRE Y DURANTE COMPETENCIA

Artigo Original

Carlos Eduardo Lopes Verardi ${ }^{1}$

(Educador Fisico)

Adriana Barbosa Santos ${ }^{3}$

(Estatística)

Kazuo Kawano Nagamine ${ }^{2}$

(Educador Fisico)

Tales de Carvalho ${ }^{4}$ (Médico)

Maria Cristina de Oliveira Santos Miyazaki² (Psicóloga)

1. Universidade Estadual Paulista (UNESP), Bauru, SP, Brasil.

2. Faculdade de Medicina de São José do Rio Preto (FAMERP), São

José do Rio Preto, SP, Brasil.

3. Departamento de Ciências de

Computação e Estatística - IBILCE,

(UNESP) - São José do Rio Preto,

SP, Brasil.

4. Universidade do Estado de Santa Catarina (UDESC), Florianópolis, SC, Brasil.

\section{Correspondencia:}

Universidade Estadual Paulista (UNESP). Campus de Bauru. Faculdade de Ciências - Depto. Educação Física. Av. Eng. Luiz Edmundo Carrijo Coube, 14-01, Vargem Limpa, Bauru, SP, Brasil, 17033-360. verardi@fc.unesp.br

\section{RESUMO}

Introdução: No contexto esportivo, a falta de estratégias adequadas de enfrentamento e a síndrome de Burnout podem ser fatores limitadores do desenvolvimento e desempenho dos atletas. Objetivo: Avaliar Burnout e formas de enfrentamento entre jogadores de futebol, profissionais e de categorias de base, durante as fases pré-competição e competição. Métodos: Em estudo prospectivo, foram avaliados 73 jogadores de futebol do sexo masculino, sendo 32 profissionais (média de idade $=22 \pm 4,14$ anos) e 41 amadores (média de idade $=17 \pm 0,94$ anos), todos integrantes de três equipes participantes do Campeonato Paulista das Séries A-1 e A-2 (categoria profissional) e Copa São Paulo de Futebol Júnior (categoria amador), Estado de São Paulo, Brasil. Para avaliar as variáveis Burnout e estratégias de enfrentamento foram utilizados os instrumentos: escala modos de enfretamento de problema (EMEP) e o questionário de Burnout para atletas (QBA). Utilizou-se estatística descritiva para análise dos dados, com nível de significância de 0,05. Resultados: Observou-se que há diferenças estatisticamente significativas entre as categorias na fase pré-competição ( $p=0,026)$, mas que não há diferenças na fase de competição $(p=0,468)$. Isso sugere que amadores e profissionais utilizam estratégias de enfrentamento distintas, que na maioria dos casos, para as duas categorias, foram focalizadas no problema. Conclusão: Durante a fase de competição os atletas com escores mais elevados de Burnout referentes à dimensão reduzido senso de realização esportiva estão associados ao modo de enfrentamento focalizado nas práticas religiosas/pensamento fantasioso.

Palavras-chave: adaptação psicológica, futebol, atletas.

\section{ABSTRACT}

Introduction: In sports, the lack of adequate coping strategies and the Burnout syndrome may limit athletes' development and performance. Objective: To assess Burnout and coping strategies among professional and youth team football players during pre-competition and competition phases. Methods: In a prospective study, 73 male football players were evaluated in the State of São Paulo, being 32 professionals (mean age $=22 \pm 4.14$ years) and 41 amateurs (mean age = 17+0.94 years), all members of three teams participating in the Championship of São Paulo, Series A-1 and A-2 (professional category), and in the São Paulo Junior Soccer Cup (amateurs). To assess the variables Burnout and coping strategies, the following instruments were used: Ways of coping problem scale (EMEP) and Burnout questionnaire for athletes (QBA). Descriptive statistics were used for data analysis, with a significance level of 0.05. Results: Statistically significant differences were found between the categories in the pre-competition ( $p=0.026)$, but no differences were found during the competition phase $(p=0.468)$. This suggests that professional and amateur football players use different coping strategies, which in most cases, for both categories, were focused on the problem. Conclusion: During the competition athletes with higher Burnout scores regarding Reduced Sense of Sporting Achievement are associated with the mode of coping focused on religious and wishful thoughts.

Keywords: adaptation, psychological, soccer, athletes.

\section{RESUMEN}

Introducción: En el contexto deportivo, la falta de estrategias adecuadas de enfrentamiento y el síndrome de Burnout pueden ser factores limitadores del desarrollo y desempeño de los atletas. Objetivo: Evaluar Burnout y formas de enfrentamiento entre jugadores de fútbol, profesionales y de categorías de base, durante las fases pre-competencia y competencia. Métodos: En estudio prospectivo, fueron evaluados 73 jugadores de fútbol del sexo masculino, siendo 32 profesionales (promedio de edad $=22 \pm 4,14$ años) y 41 amateurs (promedio de edad $=17 \pm 0,94$ años), todos integrantes de tres equipos participantes del Campeonato Paulista de las Series A-1 y A-2 (categoría profesional) y Copa São Paulo de Fútbol Junior (categoría amateur), Estado de São Paulo, Brasil. Para evaluar las variables Burnout y estrategias de enfrentamiento fueron utilizados los instrumentos: escala modos de enfrentamiento de problema (EMEP) y el cuestionario de Burnout para atletas (QBA). Se usó estadística descriptiva para análisis de los datos, con nivel de significancia de 0,05. Resultados: Se observó que hay diferencias estadísticamente significativas entre las categorías en la fase pre-competencia $(p=0,026)$, pero que no hay diferencias en la fase de competencia $(p=0,468)$. Eso sugiere que amateurs y profesionales utilizan estrategias de enfrentamiento distintas, que en la mayoría de los casos, para las dos categorías, fueron focalizadas en el problema. Conclusión: Durante la fase de competencia los atletas con escores más elevados de Burnout referentes a la dimensión Reducido Sentido de Realización Deportiva están asociados al modo de enfrentamiento focalizado en las prácticas religiosas/pensamiento fantasioso.

Palabras clave: adaptación psicológica, fútbol, atletas. 


\section{INTRODUÇÃO}

Estresse pode ser compreendido como resposta ou reação individual ao estressor, ou seja, ao estímulo ou evento ambiental causador de tensão, sendo caracterizado por manifestações psicológicas e fisiológicas que podem ser moduladas estratégias comportamentais, cognitivas e emocionais $^{1,2}$. As respostas ao estressor são influenciadas pela percepção do indivíduo, podendo uma mesma experiência proporcionar resultados individuais distintos ${ }^{3-5}$.

Habitualmente, atletas estão expostos a diferentes fontes de estresse associadas não somente ao próprio treinamento esportivo ${ }^{6}$. No contexto esportivo, a falta de estratégias adequadas de enfrentamento e a síndrome de Burnout podem se constituir em fatores limitadores para o desenvolvimento e desempenho dos atletas ${ }^{7-9}$. Enfrentamento (coping) é o termo utilizado para designar o processo de lidar com demandas (internas ou externas), avaliadas pelo indivíduo como além dos seus recursos ou suas possibilidades $^{10}$. Em atletas, Burnout é conceituado como uma resposta ao excessivo estresse crônico, sendo caracterizado por redução no desempenho, pela exaustão física e emocional, reduzido senso de realização e sentimento de desvalorização esportiva ${ }^{7,11}$. Acredita-se que os atletas de sucesso serão aqueles que melhores capacidades demonstram na gestão e no controle das situações potencialmente estressantes ${ }^{4}$, sendo esta afirmação corroborada por diversos estudos sobre estresse em atletas ${ }^{12-14}$.

Para identificar precocemente os estressores e a maior vulnerabilidade para Burnout, é necessário monitorar os atletas ao longo de uma temporada competitiva ${ }^{15}$. Cabe destacar também a necessidade de identificar as estratégias de enfrentamento utilizadas pelos atletas para lidar com o estresse ${ }^{16}$, algo que permite o delineamento de programas preventivos e de intervenção. Raedeke e Smith ${ }^{17}$ identificaram que o aumento da percepção do estresse, a satisfação com o apoio social e o modo de enfrentamento por parte dos atletas estão relacionados com os níveis de Burnout. Mas, são poucas as pesquisas que procuram relacionar propostas de estratégias de enfrentamento com as diferentes dimensões de Burnout ${ }^{18}$

Diante deste contexto, fica evidente a relevância de estudos sobre estressores que possam limitar o desenvolvimento esportivo. Tendo em vista a popularidade do futebol em nosso país, foi feita a opção de analisar as associações entre os níveis de Burnout elevados de acordo com a estratégia de enfrentamento predominante, em jogadores de futebol profissionais e amadores, durante as fases de pré-competição e competição.

\section{MÉTODOS}

Em estudo prospectivo, foram avaliados 73 jogadores de futebol do sexo masculino, sendo 32 profissionais (idade $=22 \pm 4,14$ anos) e 41 amadores (idade $=17 \pm 0,94$ anos), todos integrantes de três equipes participantes do Campeonato Paulista das Séries A-1 e A-2 (categoria profissional) e Copa São Paulo de Futebol Júnior (categoria amador), Estado de São Paulo, Brasil.

Os procedimentos da pesquisa respeitaram as normas internacionais de experimentação com humanos, sendo aprovado pelo Comitê de Ética em Pesquisa da Faculdade de Medicina de São José do Rio Preto (6109/2006). Responsáveis pelos clubes e os atletas avaliados, que assinaram previamente um Termo de Consentimento Pós-Esclarecido, também aprovaram a realização do estudo, após os devidos esclarecimentos.

Os dados foram coletados durante a fase pré-competitiva e competitiva do Campeonato Paulista da Primeira Divisão das Séries A-1 e A-2 (categoria profissional) e Copa São Paulo de Futebol Júnior (categoria amadora). Os atletas foram avaliados individualmente, sendo que aqueles que se enquadravam nos critérios de inclusão foram entrevistados "in loco", sendo avaliados no próprio local de treinamento, antes ou após a sessão de treinamento.
Para avaliar as variáveis Burnout e estratégias de enfrentamento foram utilizados os seguintes instrumentos:

1) Escala modos de enfrentamento de problema (EMEP) desenvolvida por Seidl et al. ${ }^{19}$, a partir da versão adaptada para o português ${ }^{20}$. A escala inclui 45 itens, agrupados em quatro fatores: 1) enfrentamento focalizado no problema (18 itens); 2) enfrentamento focalizado na emoção (15 itens); 3) busca de práticas religiosas (7 itens); 4) busca de suporte social (5 itens). As respostas são fornecidas em um escala do tipo Likert, com escores variando de 1 (Eu nunca faço isso) a 5 (eu sempre faço isso).

2) Questionário de Burnout para atletas $(Q B A)$, que se trata de uma versão do Athlete Burnout Questionaire $(A B Q)$, instrumento original desenvolvido especialmente para avaliar Burnout em atletas por Raedeke e Smith ${ }^{11}$, validado para o português por Pires et al. ${ }^{21}$. O questionário é composto por 15 itens e três subescalas que avaliam a frequência de sentimentos relativos ao Burnout: exaustão física e emocional (eu estou exausto pelas demandas física e emocional do esporte); reduzido senso de realização esportiva (não importa o que eu faço, eu não executo como devo); desvalorização da modalidade esportiva (eu tenho sentimentos negativos em relação ao esporte). As respostas são dadas em uma escala tipo Likert: 1) Quase nunca; 2) Raramente; 3) Algumas vezes; 4) Frequentemente; 5) Quase sempre.

\section{Análise de Dados}

Para análise da idade dos jogadores utilizou-se estatísticas descritivas como média, mediana, desvio padrão, mínimo, máximo e quartis, os quais contribuíram para se ter uma visão mais abrangente do perfil dos jogadores. Para comparar os dois grupos de jogadores quanto às proporções relativas aos fatores predominantes, utilizou-se o teste de homogeneidade qui-quadrado, tanto para a fase de pré-competição como de competição. Também foi empregado o teste qui-quadrado na avaliação do nível de Burnout em relação à estratégia de enfrentamento predominante, para as três diferentes dimensões: exaustão física e emocional (EFE), reduzido senso de realização esportiva (RSR) e desvalorização da modalidade esportiva (DES) durante a fase de pré-competição e competição. O software Minitab ${ }^{\circledR} 16$ foi utilizado nos cálculos estatísticos. Adotou-se um nível de significância de 0,05 em todos os testes de significância realizados.

\section{RESULTADOS}

O fator predominante utilizado pelo atleta para enfrentar situações problema é o que apresenta a maior média aritmética, conforme consta na tabela 1. A análise de fatores predominantes baseou-se no fator que apresentava maior média aritmética frente aos outros fatores de enfrentamento. A maior média aritmética atesta o fator predominante utilizado pelo jogador para enfrentar situações problema.

Os resultados da tabela 1 referente ao teste de homogeneidade evidenciam que há diferenças estatisticamente significativas entre as categorias na fase de pré-competição $(p=0,026)$, mas não evidenciam diferenças na fase de competição $(p=0,468)$. Isto sugere que amadores e profissionais utilizam estratégias de enfrentamento distintas, que na maioria dos casos para as duas categorias foram focalizadas no problema (fator 1). Nota-se que na fase de pré-competição para os profissionais esta estratégia (fator 1) é mais frequente do que para os amadores, sendo utilizada por $71,8 \%$ dos profissionais e 41,5\% dos amadores. As estratégias focadas em práticas religiosas (fator 3 ) e busca de suporte social (fator 4) são mais utilizadas pelos amadores (58,6\%) do que profissionais (28,2\%). O resultado da fase de pré-competição não se repete na fase de competição, embora, na maioria dos casos o modo de enfrentamento continue sendo focalizado no problema. Verifica-se um aumento do percentual dos atletas amadores que possuem o fator 1 (focalizada no problema) como predominante (cerca de $14,6 \%$ de aumento) e consequentemente 
Tabela 1. Distribuição percentual dos fatores predominantes, de acordo com a categoria e fase. Valor P referente ao teste de homogeneidade.

\begin{tabular}{c|c|c|c|c|c|c}
\hline \multirow{2}{*}{ Fase } & \multirow{2}{*}{ Categoria } & \multicolumn{3}{|c|}{ Fator predominante } & \multirow{2}{*}{ Total } & $\begin{array}{c}\text { Valor P } \\
\text { Qui-quadrado }\end{array}$ \\
\cline { 3 - 6 } & & $\mathbf{1}^{*}$ & $\mathbf{3}^{* *}$ & $\mathbf{4}^{* * *}$ & & \\
\hline $\begin{array}{c}\text { Pré- } \\
\text { competiçãon }\end{array}$ & Amador & $17(41,5 \%)$ & $12(29,3 \%)$ & $12(29,3 \%)$ & 41 & \multirow{2}{*}{0,026} \\
\cline { 2 - 6 } & Profissional & $23(71,8 \%)$ & $03(9,4 \%)$ & $06(18,8 \%)$ & 32 & \\
\hline \multirow{2}{*}{ Competição } & Amador & $23(56,1 \%)$ & $11(26,8 \%)$ & $07(17,1 \%)$ & 41 & \multirow{2}{*}{0,468} \\
\cline { 2 - 6 } & Profissional & $22(68,8 \%)$ & $05(15,6 \%)$ & $05(15,6 \%)$ & 32 & \\
\hline
\end{tabular}

*Fator 1. Estratégias de Enfrentamento Focalizadas no Problema; **Fator 3. Práticas Religiosas/Pensamento Fantasioso; ***Fator 4. Busca de Suporte Social.

uma diminuição dos que utilizam os fatores 3 e 4 (cerca de 14,7\% de diminuição). Para os atletas profissionais os percentuais dos fatores predominantes apresentaram variações discretas.

A análise dos níveis de Burnout de acordo com a estratégia de enfrentamento predominante baseou-se em observar níveis de Burnout elevados mediante respostas superiores da frequência dos sentimentos, mudando de raramente para algumas vezes, de acordo com a escala do tipo Likert de (1 a 5) e verificar a relação entre os resultados obtidos e a estratégia de enfrentamento de problemas mais utilizada por estes atletas, durante as fases de pré-competição e competição. Em relação aos sentimentos relacionados à síndrome de Burnout nas três subescalas, adotou-se para a interpretação dos escores o valor médio menor ou igual a 2,5 para indicar uma baixa frequência, sendo considerados valores elevados aqueles superiores a 2,5.

As tabelas 2 e 3 apresentam os resultados de acordo com as três dimensões de Burnout, as quais são as seguintes: exaustão física e emocional (EFE); reduzido senso de realização esportiva (RSR) e desvalorização da modalidade esportiva (DES). Durante a fase de pré-competição nota-se (tabela 2) que os atletas que apresentam escores superiores a 2,5 e não se focam em uma estratégia de enfrentamento específica. Este fato é verificado ao analisarmos os valores $p$ referentes ao teste Qui Quadrado $(p>0,05)$.

Tabela 2. Níveis de Burnout de acordo com a estratégia de enfrentamento predominante, segundo as três dimensões exaustão física e emocional (EFE); reduzido senso de realização esportiva (RSR) e desvalorização da modalidade esportiva (DES) durante a fase de pré-competição.

\begin{tabular}{|c|c|c|c|c|c|c|c|}
\hline \multirow{3}{*}{$\begin{array}{c}\text { Fator Predo- } \\
\text { minante }\end{array}$} & \multicolumn{6}{|c|}{ Dimensões Burnout } & \multirow{3}{*}{ Total } \\
\hline & \multicolumn{2}{|c|}{ EFE } & \multicolumn{2}{|c|}{\begin{tabular}{|c|} 
RSR \\
\end{tabular}} & \multicolumn{2}{|c|}{ DES } & \\
\hline & $\leq 2,5$ & $>2,5$ & $\leq 2,5$ & $>2,5$ & $\leq 2,5$ & $>2,5$ & \\
\hline Fator 1 & $38(95,0 \%)$ & $02(5,0 \%)$ & $28(70,0 \%)$ & $12(30,0 \%)$ & $30(75,0 \%)$ & $10(25,0 \%)$ & 40 \\
\hline Fator 3 & $11(73,3 \%)$ & $04(26,7 \%)$ & $09(60,0 \%)$ & $06(40,0 \%)$ & $11(73,3 \%)$ & $04(26,7 \%)$ & 15 \\
\hline Fator 4 & $16(88,9 \%)$ & $02(11,1 \%)$ & $13(72,2 \%)$ & $05(27,8 \%)$ & $13(72,2 \%)$ & $05(27,8 \%)$ & 18 \\
\hline $\begin{array}{l}\text { Valor P (teste } \\
\text { qui-quadrado) }\end{array}$ & \multicolumn{2}{|c|}{0,072} & \multicolumn{2}{|c|}{0,719} & \multicolumn{2}{|c|}{0,973} & \\
\hline
\end{tabular}

*Fator 1. Estratégias de Enfrentamento Focalizadas no Problema; **Fator 3. Práticas Religiosas/Pensamento Fantasioso; ***Fator 4. Busca de Suporte Social.

Tabela 3. Níveis de Burnout de acordo com a estratégia de enfrentamento predominante, segundo as três dimensões exaustão física e emocional (EFE); reduzido senso de realização esportiva (RSR) e desvalorização da modalidade esportiva (DES) durante a fase de competição.

\begin{tabular}{|c|c|c|c|c|c|c|c|}
\hline \multirow{3}{*}{$\begin{array}{c}\text { Fator Predo- } \\
\text { minante }\end{array}$} & \multicolumn{6}{|c|}{ Dimensões Burnout } & \multirow{3}{*}{ Total } \\
\hline & \multicolumn{2}{|c|}{ EFE } & \multicolumn{2}{|c|}{ RSR } & \multicolumn{2}{|c|}{ DES } & \\
\hline & $\leq 2,5$ & $>2,5$ & $\leq 2,5$ & $>2,5$ & $\leq 2,5$ & $>2,5$ & \\
\hline Fator 1 & $43(95,6 \%)$ & $02(4,4 \%)$ & $33(73,3 \%)$ & $12(26,7 \%)$ & $38(84,4 \%)$ & $07(15,6 \%)$ & 45 \\
\hline Fator 3 & $10(73,3 \%)$ & $06(26,7 \%)$ & $04(25,0 \%)$ & $12(75,0 \%)$ & $10(62,5 \%)$ & $06(37,5 \%)$ & 16 \\
\hline Fator 4 & $11(91,7 \%)$ & $01(8,3 \%)$ & $07(58,3 \%)$ & $05(41,7 \%)$ & $07(58,3 \%)$ & $05(41,7 \%)$ & 12 \\
\hline $\begin{array}{l}\text { Valor P (teste } \\
\text { qui-quadrado) }\end{array}$ & \multicolumn{2}{|c|}{0,002} & \multicolumn{2}{|c|}{0,003} & \multicolumn{2}{|c|}{0,071} & 73 \\
\hline
\end{tabular}

*Fator 1. Estratégias de Enfrentamento Focalizadas no Problema; **Fator 3. Práticas Religiosas/Pensamento Fantasioso; ***Fator 4. Busca de Suporte Social.
Assim, de uma maneira geral, não observamos uma relação entre o modo como os atletas enfrentam seus problemas e o nível de Burnout elevado.

Observa-se (tabela 2) que o menor percentual (5\%) referente ao nível de Burnout elevado se encontra no fator 1 (modo de enfrentamento focalizado na emoção) e o maior percentual (40\%) é encontrado no fator 3 (modo de enfrentamento relacionado a busca de práticas religiosas/pensamentos fantasiosos). A dispersão dos dados dentro de cada dimensão de Burnout sofre variações discretas, o que não nos permite afirmar que um fator específico seja determinante dos níveis elevados $(>2,5)$.

Durante a fase de competição (tabela 3) observa-se um comportamento diferente dos atletas com relação a dimensão RSR, notando assim que o fator 3 (modo de enfrentamento focado na busca de práticas religiosas/pensamentos fantasiosos) está fortemente associado ao escore de Burnout elevado.

Com relação à dimensão EFE os escores relativos ao nível de Burnout elevado $(>2,5)$ são os que apresentam os menores percentuais nos três fatores em análise, atentando para os percentuais de 4,4\% e 8,3\% (referentes aos fatores 1 e 4 respectivamente). Já a dimensão DES não apresenta valores que identifiquem a relação entre o modo de enfrentamento de problema e o nível de Burnout.

Os valores p referentes ao teste Qui-Quadrado confirmam os resultados anteriormente descritos, obtendo-se $p=0,002$ referente a dimensão EFE e $p=0,003$ referente a RSR. Já a dimensão DES apresenta $p=0,071$, valor estatisticamente não significante (tabela 3).

\section{DISCUSSÃO}

A análise de fatores predominantes consistiu em verificar, entre todos os valores calculados, qual fator apresentava maior média aritmética frente aos outros fatores de enfrentamento. Assim, a maior média aritmética atesta o fator predominante utilizado para enfrentar situações problema. Além disso, a análise dos fatores predominantes foi suportada por um teste estatístico - o teste de homogeneidade.

Nota-se, no presente estudo, diferenças estatisticamente significativas na fase de pré-competição: o fator 1 (enfrentamento focalizado no problema) é mais utilizado pelos jogadores profissionais do que pelos amadores. Além disso, os dados mostram que ambas as categorias utilizam predominantemente a estratégia de enfrentamento focalizada no problema. Este resultado é positivo, já que essa estratégia é adequada quando se pretende alterar o problema que está causando o estresse. São condutas de aproximação em relação ao estressor, desempenhadas pelos atletas, com o objetivo de solucionar o problema, lidar ou manejar a situação estressora ${ }^{19}$.

Para modificar o estressor ou adquirir habilidades para lidar com o mesmo, torna-se necessário treinar os atletas e habilitá-los a ter comportamentos como obtenção de informação, planos da pré-competição e da competição, estabelecimento de metas, habilidades de controle de tempo, solução do problema, aumento do esforço, diálogo e adesão a um programa de reabilitação de lesão 22,23 .

Durante a fase de competição, os resultados revelam que, na maioria dos casos, a estratégia focalizada no problema continuou sendo prioritária. Outro fato a ser destacado é que as estratégias focalizadas nas práticas religiosas/pensamentos fantasiosos e busca de suporte social, são menos utilizadas pelos jogadores profissionais quando comparados com os amadores. Esse resultado pode ser atribuído à experiência dos mesmos, associada ao tempo de competição. Isso sugere que os jogadores com a idade, passam a utilizar estratégias mais adequadas e efetivas para enfrentamento dos problemas ${ }^{16}$

Os atletas amadores, ao adotar estratégias focadas na busca de suporte social, demonstram maior necessidade de apoio instrumental, emocional ou de informação para enfrentar situações causadoras de estresse. Para os atletas, o suporte social diminui as incertezas durante períodos de estresse e auxilia na recuperação física e mental ${ }^{10,23}$. $O$ déficit de suporte social 
formal, por sua vez, está associado com lesões mais frequentes e recuperação mais lenta ${ }^{12,24}$. A presença de suporte social, portanto, parece ser um importante fator protetor do Burnout em atletas ${ }^{15}$.

O Burnout no contexto esportivo está frequentemente associado ao estresse, embora nem todos os atletas que apresentam estresse desenvolvam Burnout. O estresse crônico, associado com estratégias inadequadas de enfrentamento do problema, torna os atletas mais vulneráveis ao Burnout ${ }^{24,25}$.

No presente estudo, o valor médio adotado para indicar frequência elevada nos sentimentos relacionados à síndrome de Burnout, baseou-se nas propostas apresentadas por Raedeke ${ }^{7}$, Eklund e Cresswell ${ }^{26}$ e Gustafsson et al. ${ }^{27}$. Na referida proposta, a magnitude dos escores médios associados às possíveis consequências negativas do Burnout estão distribuídas de "quase nunca" para "raramente" (escore médio > 2); de "raramente" para "algumas vezes" (escore médio entre 2 e 3); de "às vezes" para "frequentemente" (escore médio entre 3 e 4) e de "frequentemente" para "maior parte do tempo" (escore médio $\geq 4$ ). Atletas que informam escores entre 3 e 4 ou mais, já apresentam sintomas e as consequências negativas associadas ao Burnout $7,11,15$. Sendo assim, o escore de referência $(2,5)$ indica um limiar de tendência de vulnerabilidade para Burnout.

Ao analisar os resultados dos níveis de Burnout de acordo com a estratégia de enfrentamento predominante durante a fase pré-competitiva, não foi observado relação entre o modo de enfrentamento e os níveis de Burnout elevado. Constatou-se que os atletas com escores superiores a 2,5 não utilizam uma estratégia de enfrentamento específica.

A hipótese que os jogadores com valores mais elevados de Burnout apresentam diferentes modos de enfrentar as situações problema foi confirmada durante a fase de competição. Os resultados apresentam comportamento contrário ao da fase de pré-competição, especialmente em relação à dimensão do Burnout relacionado ao reduzido senso de realização esportiva (RSR). Os dados sugerem que durante a fase de competição a estratégia de enfrentamento predominante dos atletas foi o fator 3 (busca de práticas religiosas/pensamentos fantasiosos), que está fortemente associado ao escore mais elevado de Burnout.

Esse resultado corrobora a afirmação que, muitas vezes, a competição é uma fonte causadora de estresse para atletas de qualquer idade e pode comprometer o seu desempenho ${ }^{14,28}$. A literatura aponta que, ao apresentar aumento dos sentimentos negativos de Burnout referente à dimensão

\section{REFERÊNCIAS}

1. Lipp MEN. Mecanismos neuropsicofisiológicos do stress: teoria e aplicações. São Paulo: Casa do Psicólogo; 2003

2. Sarafino EP. Health Psychology: biopsychosocial interactions. 6th ed. New York: John Wiley \& Sons; 2008.

3. Taylor SE. Health psychology. 7th ed. Boston: McGraw-Hill; 2009.

4. Marques ACP, Rosado AFB. Situações estressantes nos atletas de basquetebol de alta competição. Rev Bras Educ Fís Esporte. 2005;19:71-87.

5. Lazarus RS. From psychological stress to the emotions: a history of changing outlooks. Ann Rev Psychol. 1993:44(1):1-21

6. Arruda AFS, Moreira A, Nunes JA, Viveiros L, De Rose Junior D, Aoki MS. Monitoramento do nível de estresse de atletas da seleção brasileira de basquetebol feminino durante a preparação para a Copa América 2009. Rev Bras Med Esporte. 2013;19(1):44-7.

7. Raedeke TD. Is athlete burnout more than just stress? A sport commitment perspective. J Sport Exerc Psychol. 1997;19:396-417.

8. Anshel MH, Si G. Coping styles following acute stress in sport among elite Chinese athletes: a test of trait and transactional coping theories. J Sport Behav. 2008;31(1):2-21.

9. Thatcher J, Day MC. Re-appraising stress appraisals: the underlying properties of stress in sport. Psychol Sport Exerc. 2008;9:318-35.

10. Taylor SE, Stanton AL. Coping resources, coping processes, and mental health. Ann Rev Clin Psychol. 2007:3:377-401.

11. Raedeke, TD, Smith, AL. Development and preliminary validation of an athlete burnout measure. J Sport Exerc Psychol. 2001;23:238-306.

12. Ivarsson A, Urban J. Psychological factors as predictors of injuries among senior soccer players. A prospective study. J Sports Sci Med. 2010;9(2):347-352

13. Noblet AJ, Gifford S. The Sources of Stress Experienced by Professional Australian Footballers. J Appl Sport Psychol. 2002;14(1):1-13.

14. Hill AP, Appleton PR. The predictive ability of the frequency of perfectionistic cognitions, self-oriented perfectionism, and socially prescribed perfectionism in relation to symptoms of burnout in youth rugby players. J Sports Sci. 2011;29(7):695-703 do reduzido senso de realização esportiva (RSE) decorrente das demandas excessivas impostas pela competição, os atletas relatam reduzido senso de auto-realização e sucesso, com a percepção de que os objetivos inerentes ao contexto esportivo são inatingíveis ${ }^{7,27}$.

Outro aspecto associado é que os jogadores entrevistados no presente estudo, durante a fase competitiva, utilizam com maior frequência a estratégia focalizada nas práticas religiosas/pensamento fantasioso. Pode-se pressupor que esses atletas enfrentem situações problema com pensamento voltado para a fé, reduzindo assim pensamentos voltados para a situação de estresse ${ }^{19}$. Este é um dado relevante e preocupante, pois durante a competição quando a estratégia predominante é a busca de práticas religiosas/pensamentos fantasiosos combinados aos níveis elevados de Burnout, especialmente na dimensão do reduzido senso de realização esportiva, poderá haver uma auto-avaliação negativa associada a sentimentos de insatisfação, inclusive com o próprio desempenho.

Atletas que utilizam estratégias de enfrentamento inadequadas, ou que não são capazes de interpretar os acontecimentos relacionados ao contexto esportivo com racionalidade, experimentam estresse crônico e prolongado, que se traduz na redução da qualidade de execução, na vulnerabilidade ao Burnout e abandono esportivo ${ }^{29}$.

Recomenda-se, portanto, que os atletas aprendam um conjunto diferente de estratégias de enfrentamento, centradas no problema ou na emoção, para prepará-los para lidar efetivamente com as emoções nas muitas e, às vezes, novas situações de estresse. Possuir estratégias adequadas de enfrentamento permite que os atletas lidem efetivamente com os eventos imprevistos em uma competição ${ }^{23}$.

\section{CONCLUSÃO}

Durante a fase de competição os escores mais elevados de Burnout, observados em atletas que relatam com frequência a percepção de sentimentos negativos relativos ao reduzido senso de realização esportiva, estão fortemente associados com o modo de enfrentamento do problema focalizado nas práticas religiosas/pensamento fantasioso.

Todos os autores declararam não haver qualquer potencial conflito de interesses referente a este artigo.
15. Cresswell SL, Eklund RC. Changes in athlete burnout over a thirty-week "rugby year". J Sci Med Sport. 2006;9(1-2):125-34.

16. Nicholls AR, Polman RCJ. Coping in sport: a systematic review. J Sports Sci. 2007;25(1):11-31.

17. Raedeke TD, Smith AL. Coping resources and athlete Burnout: an examination of stress mediated and moderation hypotheses. J Sport Exerc Psychol. 2004;26:525-41.

18. Goodger K, Gorely T, Lavallee D, Harwood C. Burnout in sport: a systematic review. Sport Psychol. 2007;21:127-51.

19. Seidl EMF, Tróccoli BT, Zannon CML. Análise fatorial de uma medida de estratégias de enfrentamento. Psicol Teor Pesq. 2001;17:225-34

20. Gimenes MGG, Queiroz B. As diferentes fases de enfrentamento durante o primeiro ano após a mastectomia. In: Gimenes MGG, Fávero MH. A mulher e o câncer. Campinas: Editorial Psy;1997. p. 171-95.

21. Pires DA, Brandão MRF, Silva CB. Validação do questionário de burnout para atletas. Rev Educ Fís/UEM 2006;17(1):27-36.

22. Straub RO. Psicologia da Saúde. Porto Alegre: Artmed; 2005

23. Weinberg RS, Gould D. Fundamentos da psicologia do esporte e do exercício. Porto Alegre: Artmed; 2008

24. Cresswell SL, Eklund RC. Motivation and Burnout in professional rugby players. Res Q Exerc Sport 2005;76(3):370-6.

25. Cresswell SL. Possible early signs of athlete burnout: a prospective study. J Sci Med Sport. 2009;12(6):393-8. 26. Eklund RC, Cresswell SL. Athlete burnout. In: Tenenbaum G, Eklund RC. Handbook of sport psychology New York: Wiley; 2007. p. 621-41.

27. Gustafsson H, Hassme' $n$ P, Podlog L. Exploring the relationship between hope and burnout in competitive sport. J Sports Sci. 2010;28:1495-504

28. Hanton S, Thomas O, Mellalieu SD. Management of competitive stress in elite sport. In: Brewer B. International Olympic Committee Sport Psychology Handbook. New York: Blackwell; 2008. p. 30-42.

29. Márquez S. Estrategias de afrontamiento del estrés en el ámbito deportivo: fundamentos teóricos e instrumentos de evaluación. Int J Clin Health Psychol. 2006;6:359-78. 